\title{
Artificial Intelligence Algorithms for the Analysis of Mechanical Property of Friction Stir Welded Joints by using Python Programming
}

\author{
Akshansh Mishra ${ }^{\circledR}$ \\ Department of Mechanical Engineering, Politecnico Di Milano, Italy \\ *Correspondence: akshansh.frictionwelding@gmail.com
}

Received: 07.06.2020; Accepted: 12.08.2020

\begin{abstract}
In modern computational science, the interplay existing between machine learning and optimization process marks the most vital developments. Optimization plays an important role in mechanical industries because it leads to reduce in material cost, time consumption and increase in production rate. The recent work focuses on performing the optimization task on Friction Stir Welding process for obtaining the maximum Ultimate Tensile Strength (UTS) of the friction stir welded joints. Two machine learning algorithms i.e. Artificial Neural Network (ANN) and Decision Trees regression model are selected for the purpose. The input variables are Tool Rotational Speed (RPM), Tool Traverse Speed $(\mathrm{mm} / \mathrm{min})$ and Axial Force $(\mathrm{KN})$ while the output variable is Ultimate Tensile Strength (MPa). It is observed that in case of the Artificial Neural Networks the Root Mean Square Errors for training and testing sets are 0.842 and 0.808 respectively while in case of Decision Trees regression model, the training and testing sets result Root Mean Square Errors of 11.72 and 14.61. So, it can be concluded that ANN algorithm gives better and accurate result than Decision Tree regression algorithm.
\end{abstract}

Keywords: Machine Learning; Artificial Neural Network; Decision Trees; Optimization; Friction Stir Welding

\section{Introduction}

According to Andrew Ng who is the co-founder of Google Brain, Artificial Intelligence (AI) is going to revolutionise the manufacturing and materials industries. AI finds application in quality checks, prediction of failure modes, generative design and forecasting output for the available dataset [1-2]. AI can be defined as a simulation of human intelligence which possesses the skills of identifying, observing, learning, performing the task and if needed, it can self-correct itself [3-4]. AI can be achieved by various numbers of ways as shown in the Fig.1. It can be seen that areas like machine learning, machine vision, natural language processing, robotics etc. contributes to the formation of AI [5]. From Fig.2 it is observed that machine learning field makes an important contribution for the development of AI.

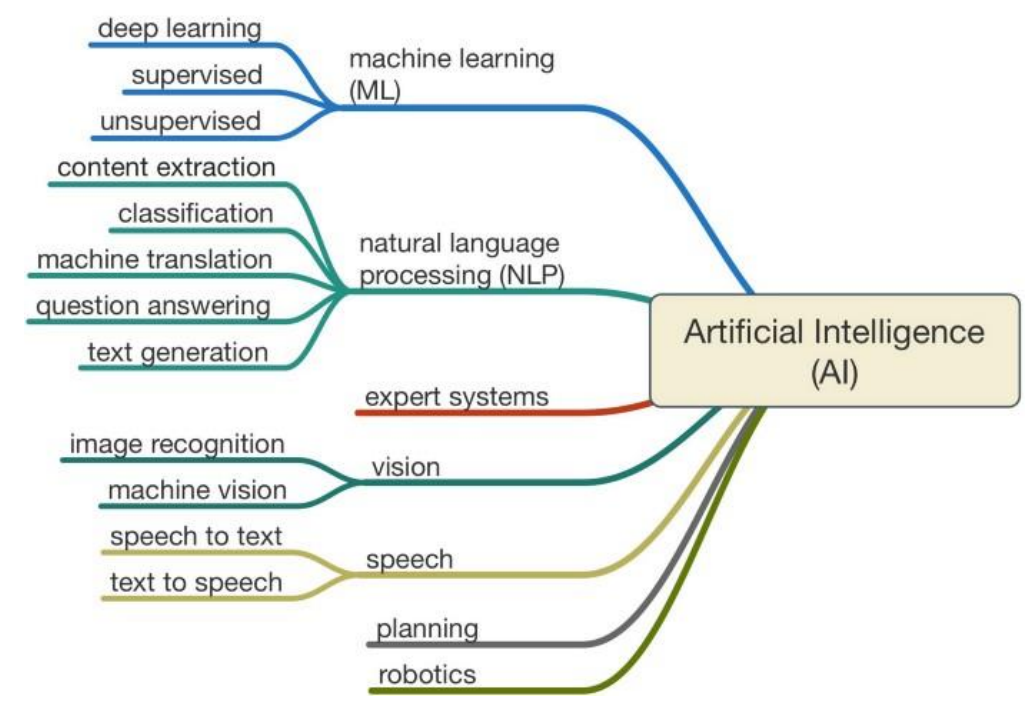

Fig. 1. Representation of number of ways AI can be achieved [6] 


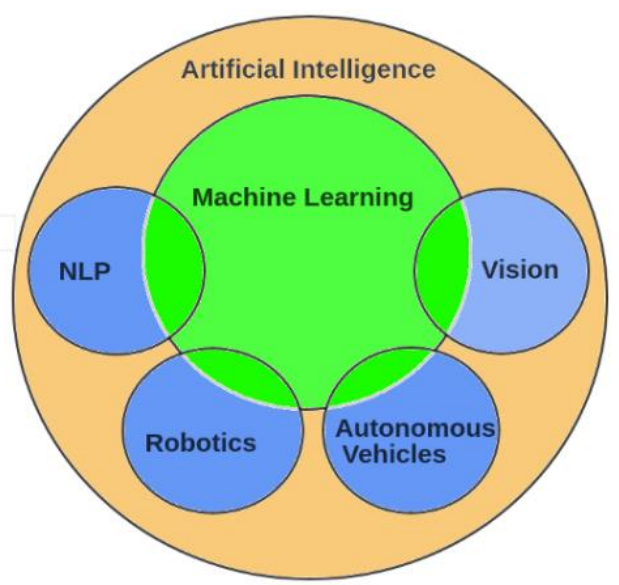

Fig. 2. Classification architecture of AI [6]

Machine learning aims to design, understand, and apply computer programs that learn from the past experience i.e. data for the purpose of modelling, prediction or control. Machine learning finds application in the development of new type of material, also it involves high-throughput density functional theory for the calculations of materials properties [7].

Lu et al. [8] worked on the development of a general machine learning framework for the extraction of elastoplastic properties of engineering alloys with strikingly improved training efficiency and accuracy. Lilienfeld et al. [9] introduced machine learning models for a sample prediction for proton and forces on atoms, excitations at atomic core level and carbon nuclear chemical shifts. Machine Learning finds application in Friction Stir Welding process which is a solid-state welding technology. Friction Stir Welding process consists of base alloy plates to be joined, welding tool whose material is harder than the alloy plates to be joined and a fixture design for holding the work piece together. In the present work, two machine learning algorithms i.e. Artificial Neural Network and Decision Tree regression model have been used for the prediction of Ultimate Tensile Strength (UTS) of friction stir welded joints 6061-T6 aluminium alloy joints.

\section{Working principle of Artificial Neural Network (ANN) and Decision Tree Regression}

Perceptron is the simplest neuron which consists of an output layer and an input layer. This neuron contains two types of functions i.e. summation function and activation function. It is observed from the Fig. 3 shows that the summation function processes the inputs incorporated into the perceptron and then it is further subjected to the activation function in order to get the output.

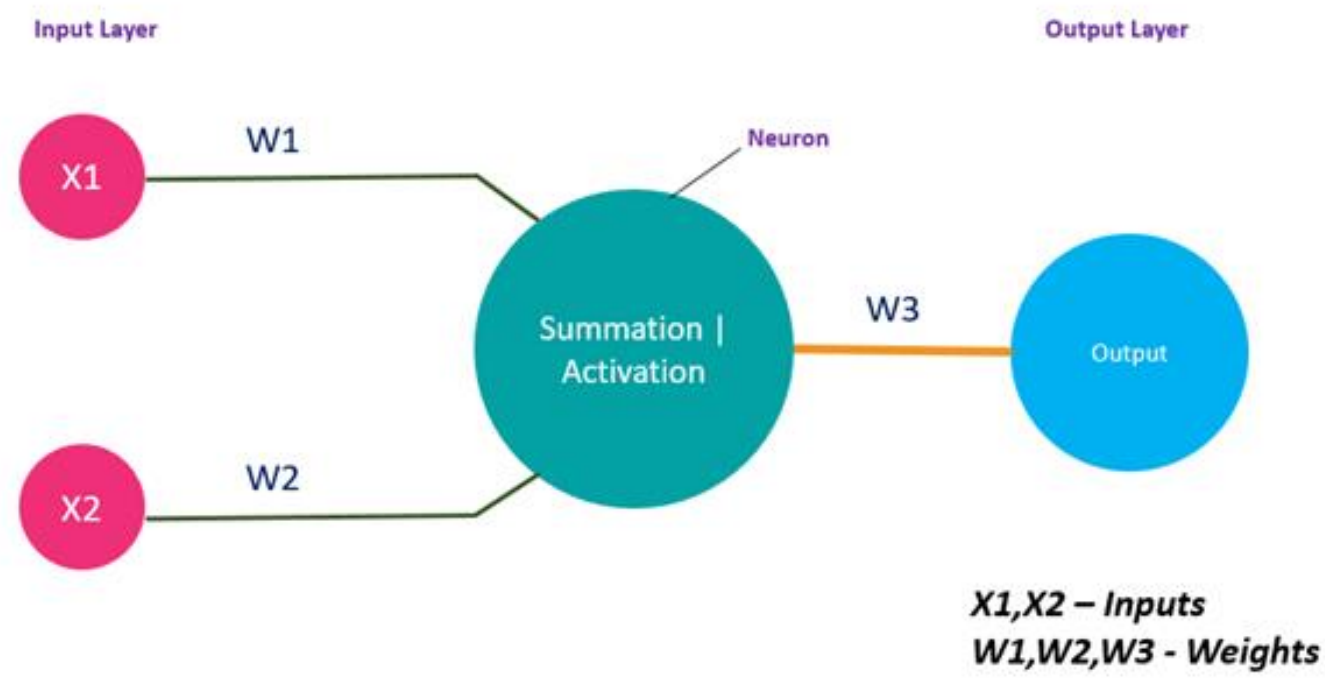

Fig. 3. Design of Simple Perceptron model [10] 
The main disadvantage of simple perceptron model is its incapability to process huge datasets and multiple inputs. In order to overcome this issue, we should keep increasing the number of neurons. Fig. 4. shows a basic artificial neural network architecture consisting of an output layer, hidden layers and an input layer. It should be noted that the artificial neural network can have multiple hidden layers, but it should have single output layer and input layer. The main objective of the activation function is to achieve an output by the conversion of the weighted sum of input signals.

Input Layer Hidden Layers Output Layer

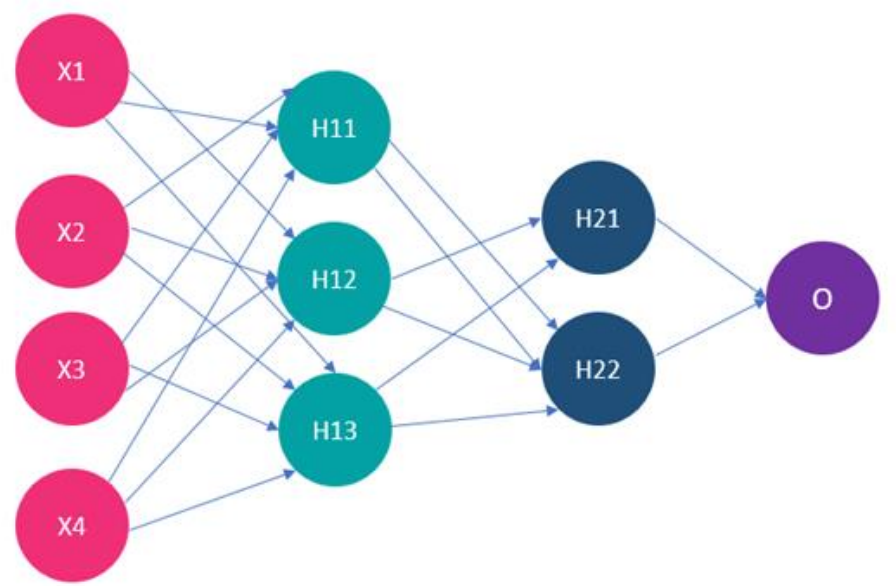

Fig.4. Architecture of Artificial Neural Network [10]

The working principle of an Artificial Neural Network is generally based on the two types of propagation methods i.e. forward propagation and backward propagation. Fig. 5. explains the working principle of forward propagation method.

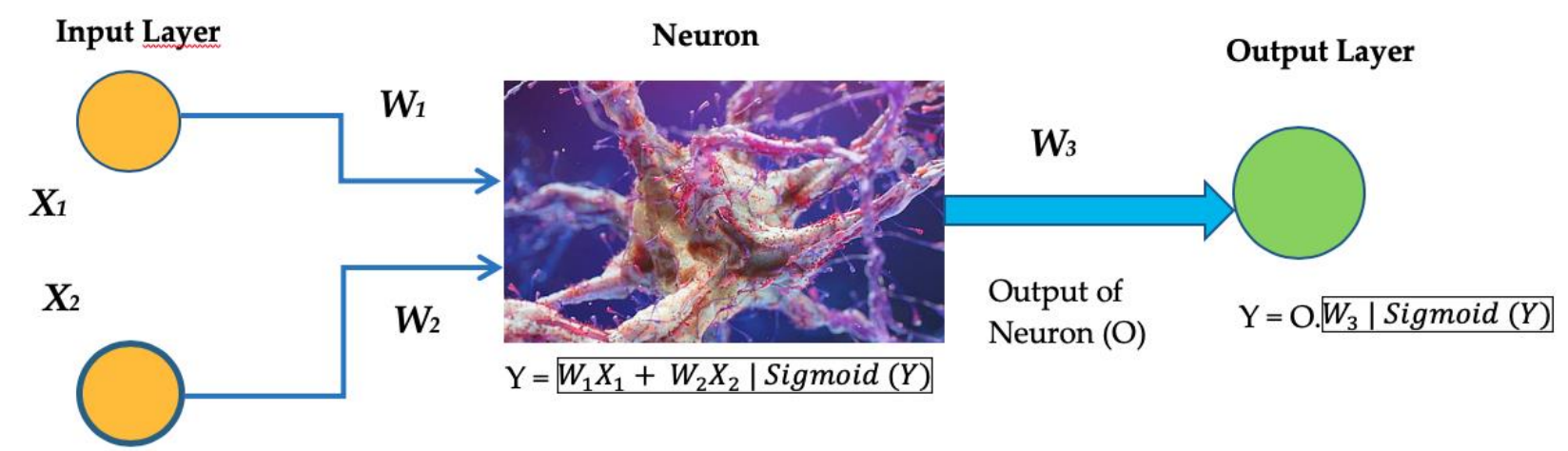

Fig. 5. Working principle of Forward Propagation method

$X_{1}$ and $X_{2}$ are the features of a sample in a given dataset. In order to predict the outcome, these features are subjected to some mathematical operations. $W_{1}$ and $W_{2}$ are the weights which are associated with each features. These weights together serve the neuron as an input. At the summation stage, all available features are multiplied by their alotted weights and a bias is added further as shown in the equation 1.

$$
\mathrm{Y}=\overline{W_{1} X_{1}+W_{2} X_{2}+\text { bias }}
$$

Activation function is further subjected to the above summed up function. Weight $W_{3}$ is multiplied with the output of the former neuron and it further behaves as the input for an output layer. The backpropagation method is used to update the weights after each initiation. Fig. 6. shows the architecture of the backpropagation method. The main objective is to make the error minimum which can be achieved by finding the changing in weights and also calculating $\frac{d E}{d W_{1}}$ and $\frac{d E}{d W_{3}}$. 


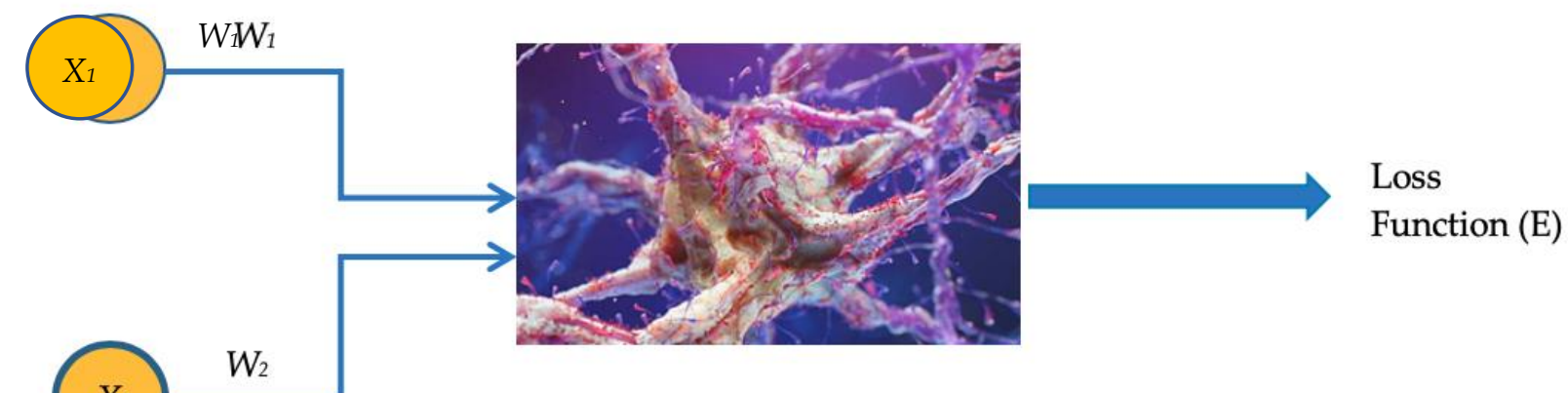

\section{Summation I Activation}

Fig. 6. Working principle of backward propagation method

$$
\begin{aligned}
& \text { Summation }(S)=\sqrt{W_{1} X_{1}+W_{2} X_{2}} \\
& \text { Activation = sigmoid }=\frac{e^{x}}{\left(1+e^{x}\right)}
\end{aligned}
$$

By using chain rule, we can find change in weights,

$$
\begin{aligned}
& \frac{d E}{d W_{1}}=\frac{d E}{d A} X \frac{d A}{d S} X \frac{d S}{d W_{1}} \\
& \frac{d E}{d W_{7}}=\frac{d E}{d A} X \frac{d A}{d S} X \frac{d S}{d W_{7}}
\end{aligned}
$$

Senthilnathan et al. [11] used Artificial Neural Network for predicting the micro hardness and tensile strength of friction stir welded dissimilar aluminium alloy plates AA 6063-O and AA 2014-T6. Mishra et al. [12] predicted the corrosion potential of friction stir welded joints by using back propagated artificial neural network. Hartl et al. [13] used artificial neural networks for the surface quality of friction stir welded joints. Rehim et al. [14] predicted and simulated the Vickers hardness of friction stir welded joints by using artificial neural network. From these researches it is observed that Artificial Neural Network has a wide application in Friction Stir Welding process.

Decision Tree regression algorithm is a non-continuous model which falls into non-linear regression category. It can be thought of as a supervised learning method which is non-parametric in nature and can be both used for regression and classification. Decision tree operates on if-then- else decision rules which learn from the given dataset and do better approximation. It should be noted that deeper the decision tree is the more fit model we get. The regression models in the decision tree are in the form of a tree structure. Fig. 7 shows the working criteria of decision tree algorithm.

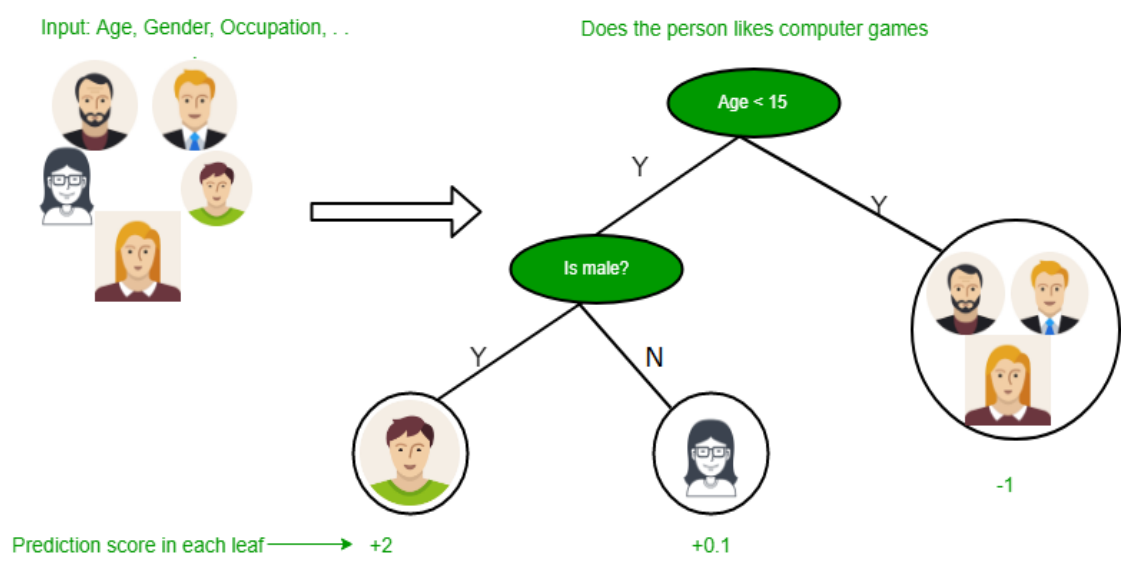

Fig. 7. Schematic representation of decision tree [15]

Bozkurt et al. [16] used decision tree regression algorithm for the prediction of the tensile property of friction stir welded AA2124/SiC/25p metal matrix composite (MMC) plates. It was observed that the decision tree algorithm can be applied with a greater accuracy. Du et al. [17] investigated about the condition of void formation using decision tree classification model and artificial neural network. It was concluded that both Artificial Neural Network and decision tree model were able to predict the void 
formation with $96.6 \%$ accuracy. In the next section, the experimental procedure dealing with the present work will be discussed.

\section{Experimental Procedure}

Firstly, 85 datasets were prepared from the previous research carried out on the friction stir welded 6061-T6 aluminium alloys [18-21]. In the prepared dataset, tool rotational speed (rpm), tool traverse speed $(\mathrm{mm} / \mathrm{min})$ and axial force $(\mathrm{KN})$ are the input variables while the Ultimate Tensile Strength $(\mathrm{MPa})$ is the output variable. Secondly, the Python programming is used for developing the code for the execution of Artificial Neural Network and Decision Tree regression algorithm. Fig. 8. shows the methodology for the Artificial Neural Network.

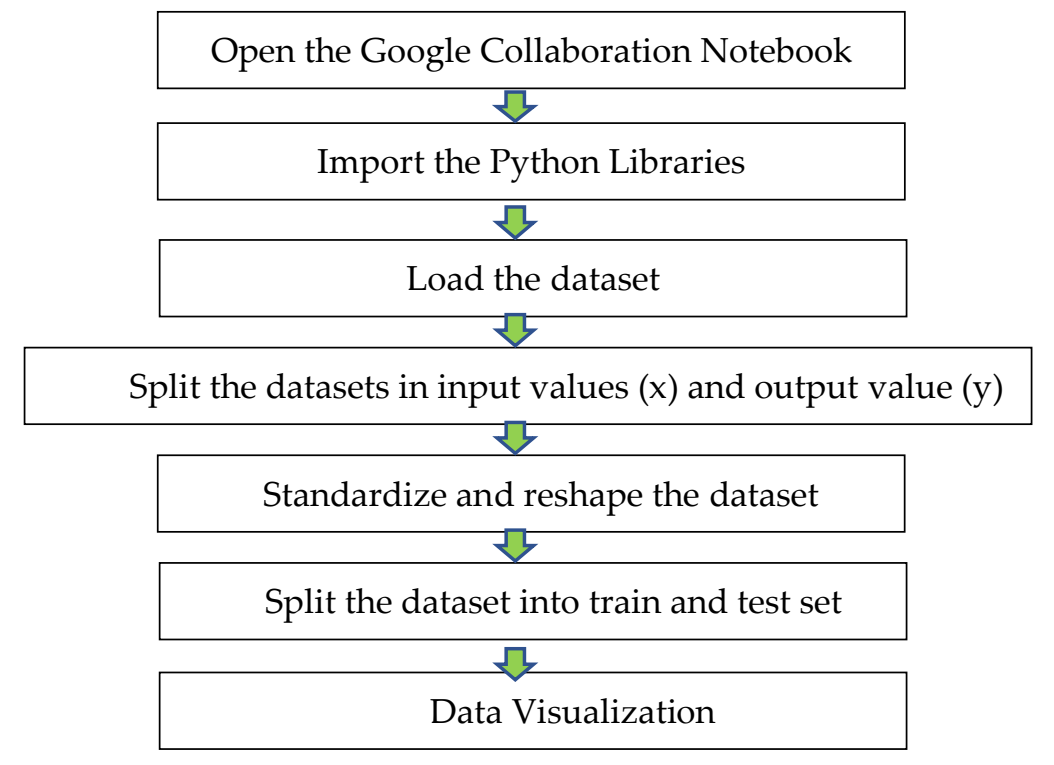

Fig. 8. Dataset subjected to Artificial Neural Network algorithm

Tool rotational speed ranged between $500 \mathrm{rpm}$ to $2000 \mathrm{rpm}$ and on other hand tool traverse speed ranged between $20 \mathrm{~mm} / \mathrm{min}$ to $150 \mathrm{~mm} / \mathrm{min}$. Fig. 9 shows the Artificial Neural Network architecture used in the present study. The input layer consisting of tool rotational speed (rpm), tool traverse speed $(\mathrm{mm} / \mathrm{min})$ and axial force $(\mathrm{KN})$ constitute the input nodes. The first hidden layer has 4 nodes which is subjected to ReLu activation function, the second hidden layer has 3 nodes which is also subjected to ReLu activation function and the output layer has one node i.e. Ultimate Tensile Strength which is subjected to sigmoid activation function. The training was performed on 63 datasets and test was performed on 22 sample datasets.

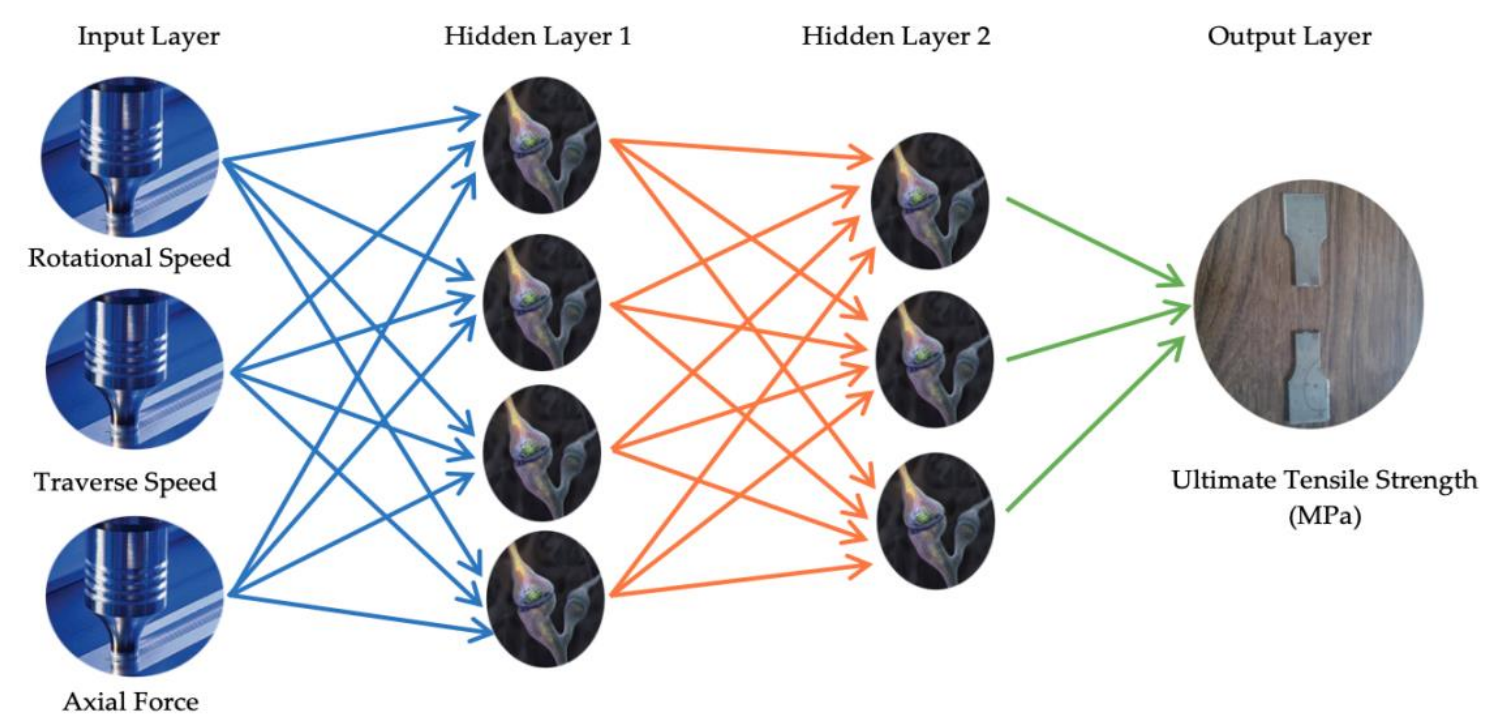

Fig. 9. Artificial Neural Network architecture for the prediction of UTS of friction stir welded alloy 
For subjecting the dataset to decision tree regression, firstly the python libraries like Numpy, Matplotlib and Pandas were imported. Secondly, the datasets were imported and the whole dataset was trained on decision tree regression model. Thirdly, the new result was predicted, and the results were visualised.

\section{Results and Discussion}

The Artificial Neural Network (ANN) model was fit with stochastic gradient descent with a learning rate of 0.01 and momentum of 0.9. Dataset training was performed for 800 epochs and test set was evaluated at the end of each epoch. Fig. 10 shows the plot of loss function for both training and testing dataset. It is observed that the loss function for both testing and training set decreases with increase in number of epochs. Fig. 11 shows the contour plot obtained between the input variables i.e. Tool traverse speed $(\mathrm{mm} / \mathrm{min})$, the tool rotational speed $(\mathrm{rpm})$ and the output variable i.e. Ultimate Tensile Strength (MPa). Fig. 12 shows the contour plot obtained between the input variables i.e. axial force $(\mathrm{KN})$, tool rotational speed (rpm) and the output variable i.e. Ultimate Tensile Strength (MPa). Fig. 13 shows the contour plot obtained between in the input variables i.e. axial force $(\mathrm{KN})$, tool traverse speed $(\mathrm{mm} / \mathrm{min})$ and the output variable i.e. Ultimate Tensile Strength (MPa). After the end of epoch, it is observed that for the Artificial Neural Networks, the Root Mean Square Errors for training and testing sets are 0.842 and 0.808.

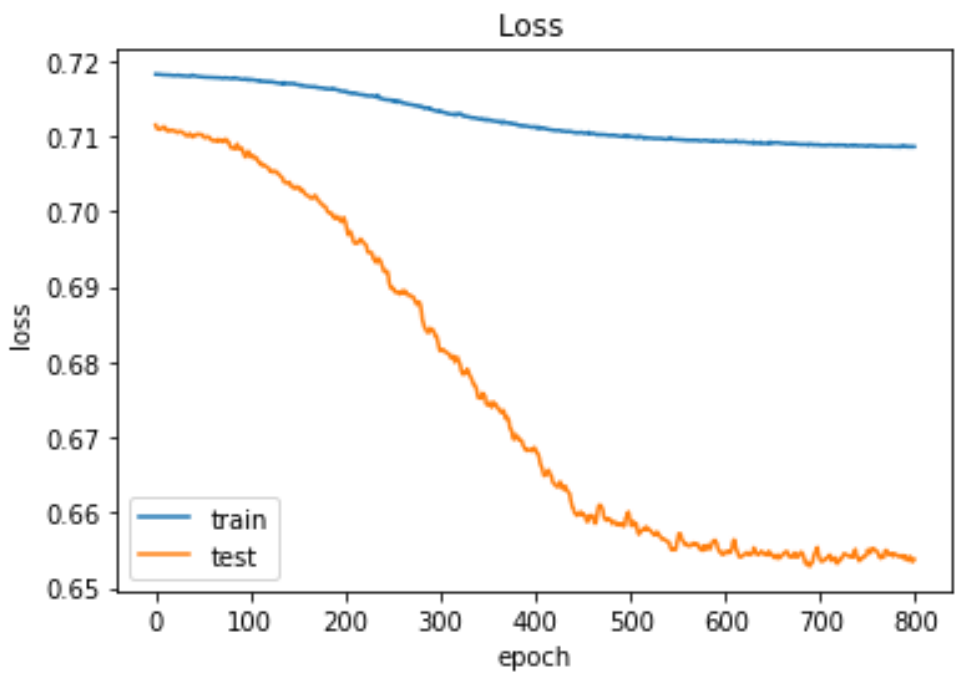

Fig. 10. Plot of loss function wrt epoch

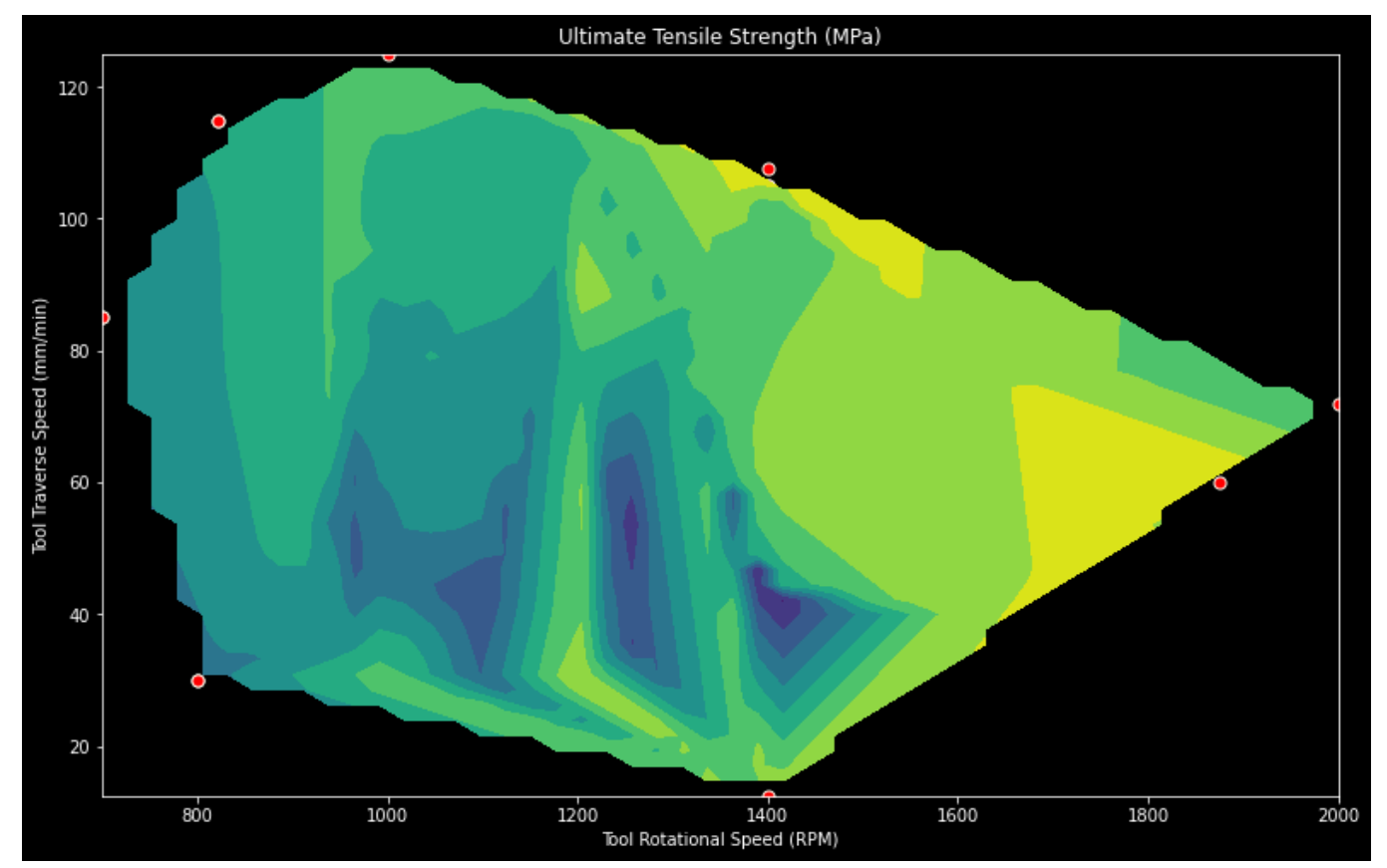

Fig. 11. Contour plot of UTS wrt Tool Traverse Speed (mm/min) and Tool Rotational Speed (rpm) 


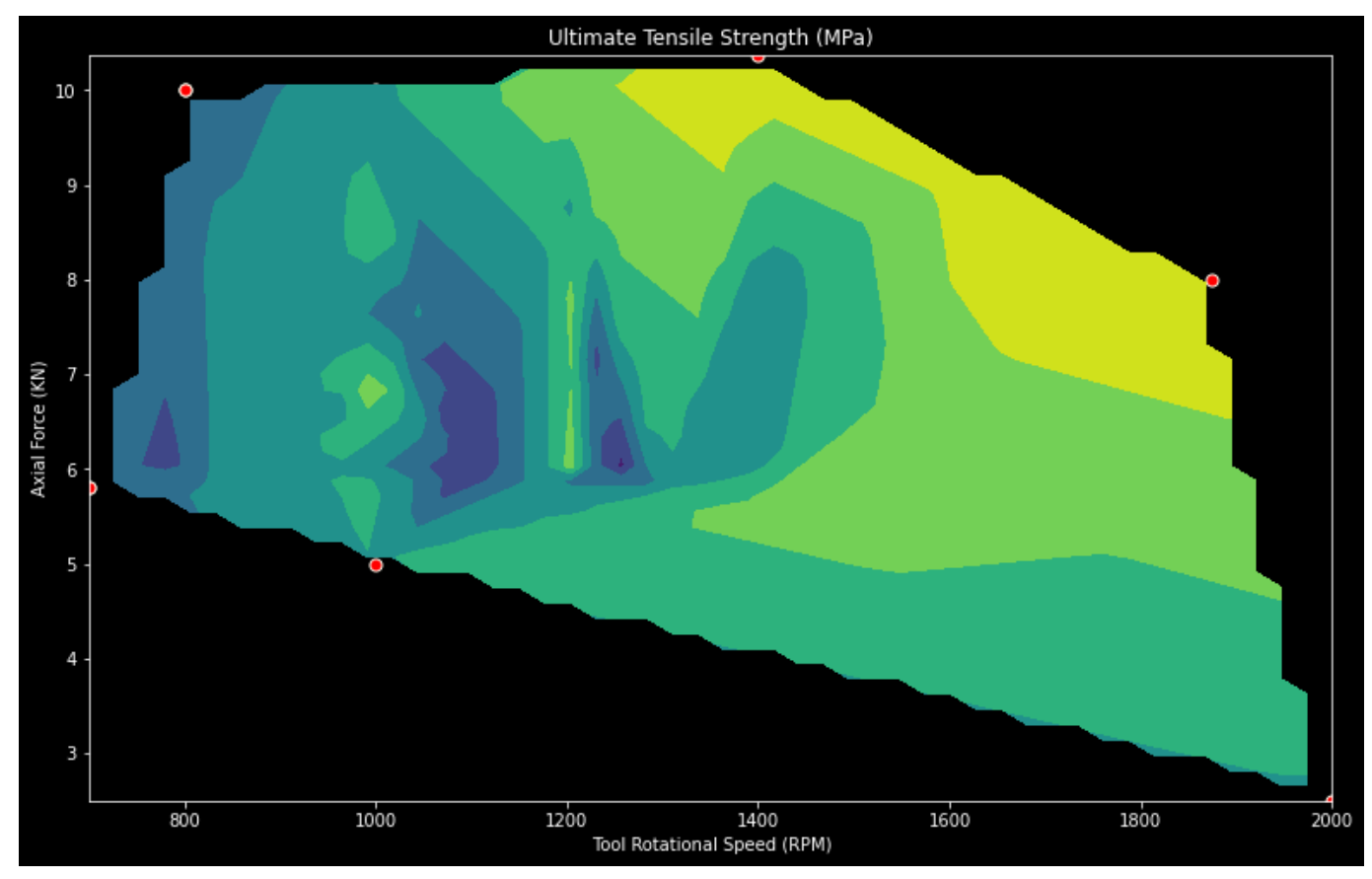

Fig. 12. Contour plot of UTS wrt Axial Force (KN) and Tool Rotational Speed (rpm)

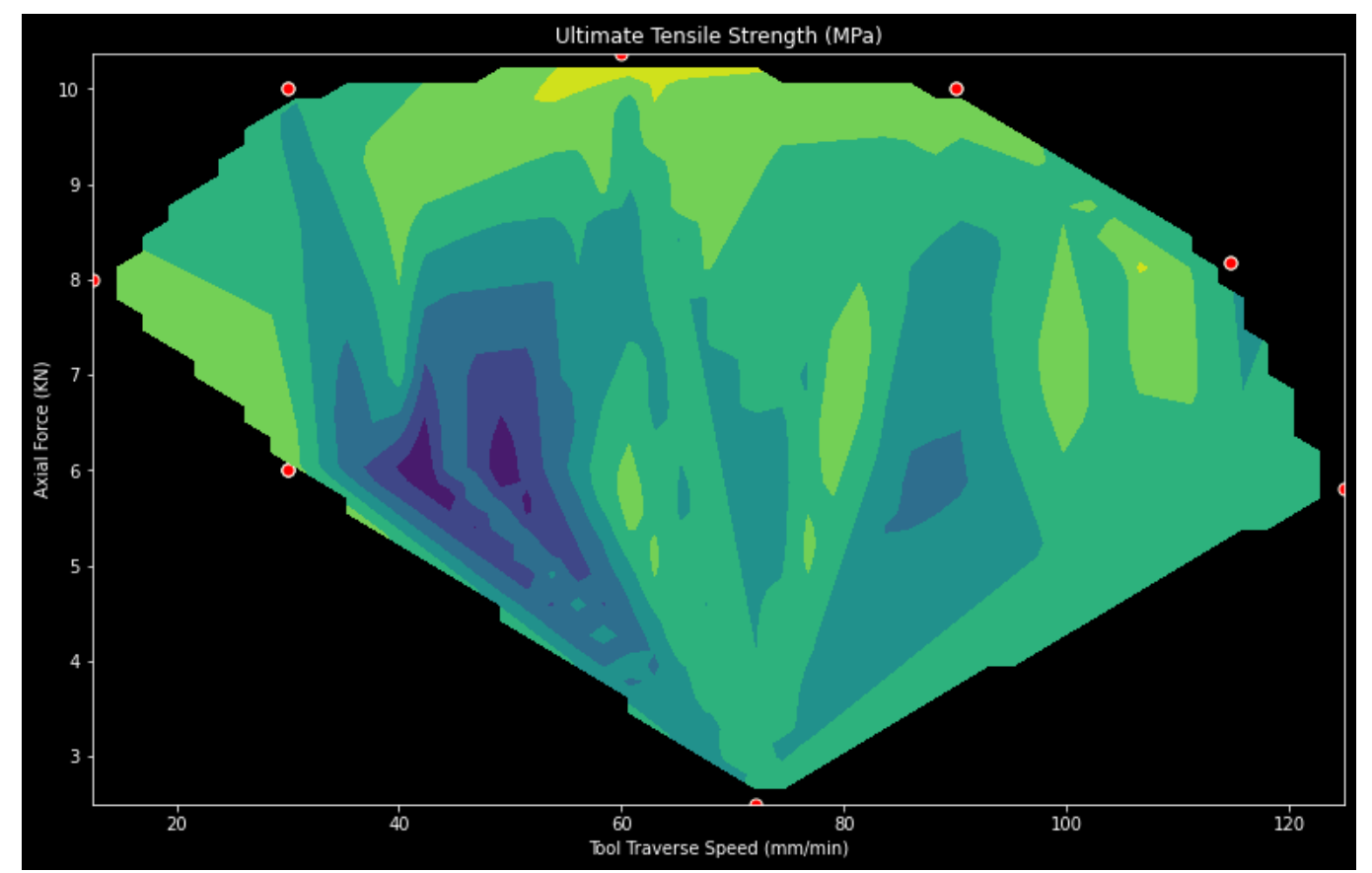

Fig. 13. Contour plot of UTS wrt Axial Force $(\mathrm{KN})$ and Tool Traverse Speed $(\mathrm{mm} / \mathrm{min})$

Decision trees regression model are bit more complex than classification tree model. Once we execute the regression tree algorithm, the scatter plot obtained will be split into many segments as shown in the Fig. 14 and Fig. 15. An algorithm called Information Entropy governs how and where these splits are conducted. So, it basically means that when the split is performed it is checked whether the split is increasing the amount of information that we have about our points or is it actually it is adding some value we want to group our points. The algorithm can well handle the optimal splits of the dataset into these leaves and the final leaves are called terminal leaves. 


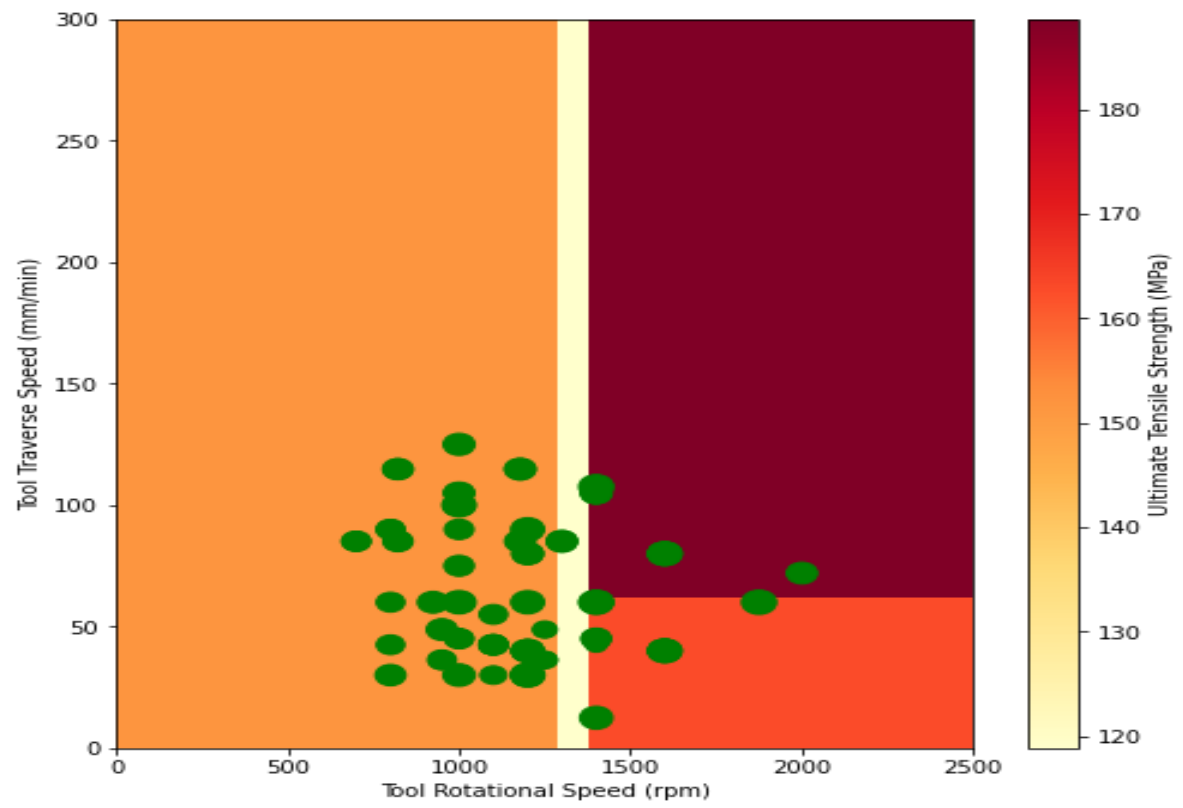

Fig. 14. Decision Tress Regression plot between UTS and Tool traverse speed (y-axis), Rotational Speed (x-axis)

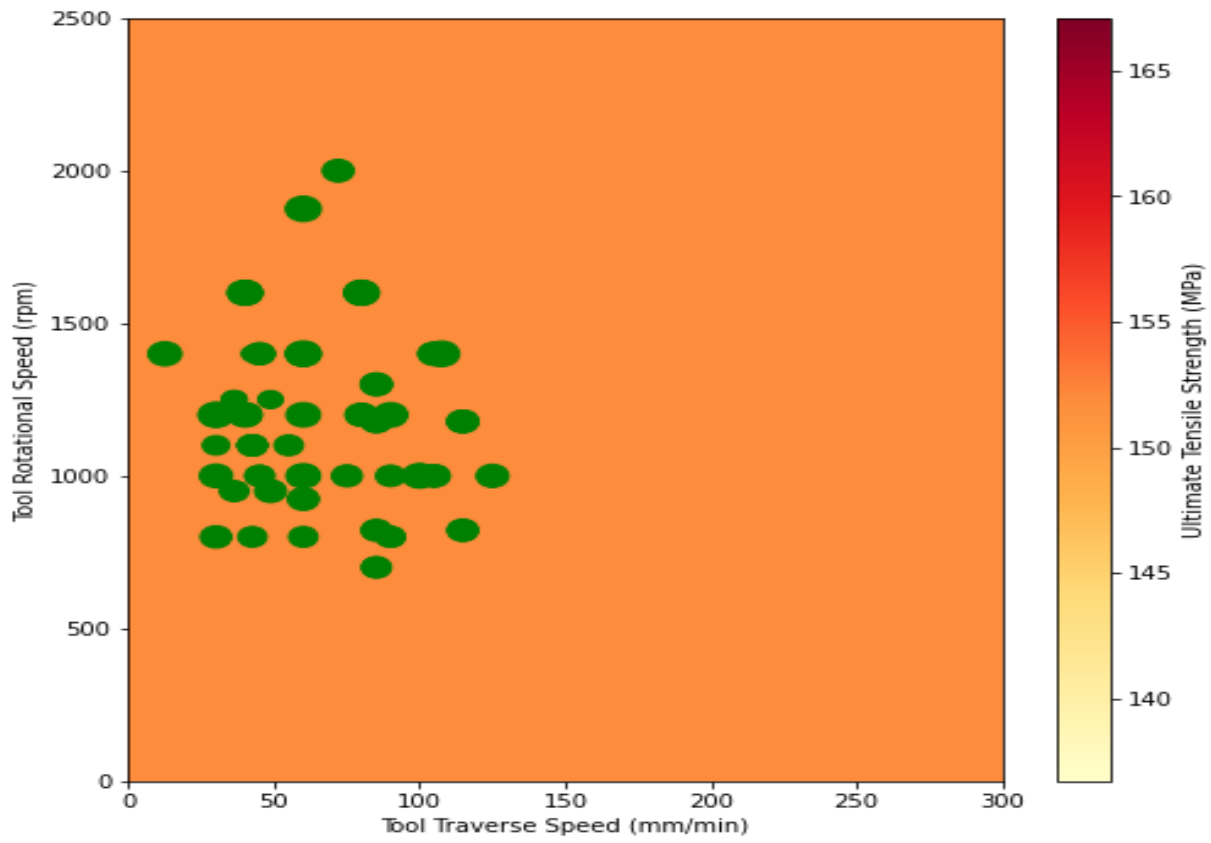

Fig.15. Decision Tress Regression plot between UTS and Tool traverse speed (x-axis), Rotational Speed (y-axis)

It is observed that the training and testing sets in decision tree regression algorithm result Root Mean Square Errors of 11.72 and 14.61.

\section{Conclusion}

Machine Learning applications in materials and manufacturing industries are found to be ground breaking. The following conclusions can be drawn from the present work:

- Artificial algorithms i.e. Artificial Neural Network (ANN) and decision tree regression models are successfully implemented in the present work.

- It is observed that in ANN, loss function decreases continuously with the increase in number of epochs.

- It is also observed that the root means square error obtained in ANN is far more less than those obtained by implementing decision tree regression model.

- The accuracy of both of the algorithms can be achieved if the number of data set is increased.

- The cost and time of the experiment is further reduced by implementing machine learning techniques. 
Funding: This research received no external funding.

Conflicts of Interest: The author declares no conflict of interest.

\section{References}

[1] Russell S.J., Norvig P., Artificial Intelligence: A Modern ApproachRussell, S. J., \& Norvig, P. (2010). Artificial Intelligence: A Modern Approach. https://doi.org/10.1017/S0269888900007724.

[2] Fogel D.B., Defining Artificial Intelligence.In: Evolutionary Computation, 2006.

[3] Ramos C., Augusto J.C., Shapiro D., Ambient intelligencethe next step for artificial intelligence. IEEE Intelligent Systems, 2008, Vol. 23(2), 15-8. https://doi.org/10.1109/MIS.2008.19

[4] Ghahramani Z., Probabilistic machine learning and artificial intelligence. Nature, 2015, Vol. 521, 4529. https://doi.org/10.1038/nature14541

[5] Ng A., What artificial intelligence can and can't do right now. Harvard Business Review Digital Articles, 2016,(2-4).

[6] Chethan K.G., Artificial Intelligence: Definition, Types, Examples, Technologies.https://doi.org/https://medium.com/@chethankumargn/artificial-intelligencedefinition-types-examples-technologies-962ea75c7b9b

[7] Meredig B., Five High-Impact Research Areas in Machine Learning for Materials Science. Chem Mater, 2019, Vol. 31(23), 9579-81. https://doi.org/10.1021/acs.chemmater.9b04078

[8] Lu L., Dao M., Kumar P., Ramamurty U., Karniadakis G.E., Suresh S., Extraction of mechanical properties of materials through deep learning from instrumented indentation. Proceedings of the National Academy of Science of the USA, 2020, Vol. 117(13), 7052-62.

https://doi.org/10.1073/pnas.1922210117

[9] Rupp M., Ramakrishnan R., Von Lilienfeld O.A., Machine Learning for Quantum Mechanical Properties of Atoms in Molecules. Journal of Physical Chemistry Letters, 2015, Vol. 6(16), 3309-13. https://doi.org/10.1021/acs.jpclett.5b01456

[10] Gajawada S.K., The Math behind Artificial Neural Networks.2019. https://doi.org/https://towardsdatascience.com/the-heart-of-artificial-neural-networks-26627e8c03ba

[11] Senthilnathan T., Sujay Aadithya B., Balachandar K., Prediction of mechanical properties and optimization of process parameters in friction-stir-welded dissimilar aluminium alloys. World Journal of Engineering, 2020, Vol. 17(4), 519-26.

[12] Mishra A., Tiwari A., Dubey N.K., Machine Learning Approach to Determine Corrosion Potential of Friction Stir Welded Joints. Journal of Image Processing \& Pattern Recognition Progress, 2020, Vol. 7(1), 5-17.

[13] Hartl R., Praehofer B., Zaeh M.F., Prediction of the surface quality of friction stir welds by the analysis of process data using Artificial Neural Networks. Proceedings of the Institution of Mechanical Engineers, Part L: Journal of Materials: Design and Applications, 2020, Vol. 234(5), 732-51. https://doi.org/10.1177/1464420719899685

[14] Abd El-Rehim A.F., Zahran H.Y., Habashy D.M., Al-Masoud H.M., Simulation and prediction of the vickers hardness of AZ91 magnesium alloy using artificial neural network model. Crystals, 2020, Vol. 10(4), 290. https://doi.org/10.3390/cryst10040290

[15] Decision Tree Introduction with example. https://doi.org/https://www.geeksforgeeks.org/decisiontree-introduction-example/

[16] Bozkurt Y., Kentli A., Uzun H., Salman S., Experimental Investigation and Prediction of Mechanical Properties of Friction Stir Welded Aluminium Metal Matrix Composite Plates. MATERIALS SCIENCE (MEDŽIAGOTYRA), 2012, Vol. 18(4), 336-40. https://doi.org/10.5755/j01.ms.18.4.3092

[17] Du Y., Mukherjee T., DebRoy T., Conditions for void formation in friction stir welding from machine learning. npj Comput Mater, 2019, Vol. 5, 68. https://doi.org/10.1038/s41524-019-0207-y

[18] Hema P., Experimental Investigations on AA 6061 Alloy Welded Joints by Friction Stir Welding. In: Cooke KO, editor. Aluminium Alloys and Composites, IntechOpen Limited, 2019. https://doi.org/10.5772/intechopen.89797

[19] Elatharasan G., Kumar V.S.S., An experimental analysis and optimization of process parameter on friction stir welding of AA 6061-T6 aluminum alloy using RSM. Procedia Engineering, 2013, Vol. 64, 1227-34. https://doi.org/10.1016/j.proeng.2013.09.202

[20] Netto N., Tiryakioglu M., Eason P., Characterization of Tool Degradation during Friction Stir 
Processing of 6061-T6 Aluminum Alloy Extrusions. Preprints, 2018, 2018080286. https://doi.org/10.20944/preprints201808.0286.v1

[21] Amirabadi H., Bani Mostafa Arab N., Safi S.V., A Novel Approach for Improving Mechanical

Properties in Friction Stir Butt Welded AA6061 Aluminum Plates by Using Preheat. Preprints, 2020, 2020020183.

(C) 2020 by the authors. Submitted for possible open access publication under the terms and conditions of the Creative Commons Attribution (CC BY) license (http://creativecommons.org/licenses/by/4.0/). 